\title{
Integrated Saturation Weighting based Color Cat Algorithm
}

\author{
Karamjit Kaur Dhillon \\ M-tech student \\ C.S.E Department \\ Amritsar College of Engineering and Technology \\ Amritsar.(India)
}

\author{
Aarti Vaish \\ Asst.Professor \\ C.S.E Department \\ Amritsar college of Engineering and Technology \\ Amritsar. (India)
}

\begin{abstract}
Color Constancy has capability to displace the precise shades in provided picture by considering the effectation of color gentle source. Many color constancy methods has been proposed so far to boost the color constancy accuracy charge further. In present literature number this type of process can be acquired which works optimistically in many case. Although the color cat shows successful benefits around available methods, however it is still struggling with the matter of irregular illuminate and bad brightness. Thus to cope with this problem for the reason that paper a brand new integrated color cat approach is proposed for the reason that dissertation. The new approach has applied color normalization and saturation weighting as post managing of color cat to lessen the effectation of irregular illuminate and bad brightness. The general gain shows the effectiveness of the proposed technique.
\end{abstract}

\section{Keywords}

Color constancy, Contrast Enhancement, Image enhancement, Adaptive Histogram Equalization method, Color and Illumination Distribution.

\section{INTRODUCTION}

Color of a pixel in the image can be described as a merchandise of 2 quantities: reflectance (the color from the paint from the surfaces within the scene) as well as illumination (the color from the light striking the surfaces within the scene). Every time a person stands inside a room lit by a colorful light-weight they unconsciously "discount this illuminant", within the words regarding Helmholtz [1], and see the objects within the room as though they had been illuminated by a neutral, whitened light. Endowing some type of computer with the identical ability is usually difficult, as this issue is mainly under constrained - presented a yellow pixel, how can discern when it is a whitened object within a yellow illuminant, or even a yellow object under a white illuminant? Essentially the most general characterization of the problem is the "intrinsic image" dilemma [2], but the precise problem regarding inferring as well as correcting the color of this illumination of your image is frequently termed as "color constancy" or perhaps "white balance".

Large of Color constancy is really a well researched in both vision science and computer system vision, as it relates to the academic study regarding human perception together with practical problems such as designing a object identification algorithm or possibly a camera. Almost all algorithms just for this task do the job by supposing some regularity within the colors regarding natural physical objects viewed beneath a whitened light.

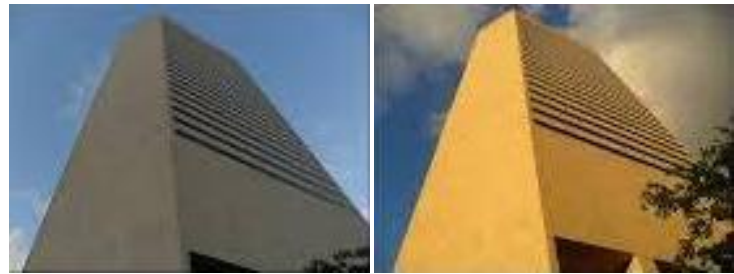

Fig: 1 Color constancy

The easiest such algorithm can be "gray world", which assumes how the illuminant color will be the average color of all image pixels, thereby implicitly assuming that object reflectance tend to be, on normal, gray. This easy idea may be generalized in order to modeling gradient details or employing generalized norms rather than a easy arithmetic necessarily mean, modeling your spatial submitting of colors that has a filter standard bank [3], modeling your distribution regarding color histograms, or implicitly reasoning about the moments regarding colors employing PCA. Other designs assume how the colors regarding natural physical objects lie having some range [4]. These types of models may be often considered as statistical, as they either believe some submitting of shades or these people learn a few distribution regarding colors via training files. This link with learning and statistics might be made much more explicit, often in a very Bayesian construction [5]. One thing these statistical or perhaps learning-based designs have in accordance is they are all generative types of natural shades - some sort of model can be learned via (or assumed of) white-balanced graphics, and subsequently that model is utilized to white-balance brand new images. On this paper, the beneath assumption will be operated probably in which white-balancing is really a discriminative job. That can be, instead regarding training some sort of generative design to delegate high likelihoods in order to white-balanced images beneath assumption that this type of model will succeed at white-balancing, a model will be probably educated explicitly to distinguish between whitebalanced graphics and non-white balanced graphics. This using discriminative device learning is apparently largely unexplored throughout context regarding color credibility, though equivalent tools happen to be used in order to augment generative shade constancy designs with encounter detection [6] or perhaps scene group details. The nearly all related strategy might be that regarding Finlayson when a simple "correction" into a generalized gray-world protocol is realized using iterative least-squares, producing state-of-the-art results in comparison with prior art.

\section{CONTRAST ENHANCEMENT}

Image form a contrast enhancement [7] and is really a classical difficulty in photograph processing and computer imaginative and prescient vision. Image enhancement is 
known as some sort of preprocessing part of many locations like video/image control applications and talks recognition, texture synthesis etc. Enhancement strategies mainly belong to two wide-ranging categories: spatial site methods and frequency site methods. Spatial site techniques are definitely more popular versus frequency primarily based methods, as they are based about direct adjustment of pixels within the image. Myriad spatial site methods happen to be developed regarding visualizing the result. Some of such methods makes use of simple linear or perhaps non-linear power level transformation functions, whereas other people use difficult analysis regarding different photograph features including the edge and connected component information. Contrast development problem throughout digital images may be resolved employing various techniques, but Histogram Equalization (HE) [8] technique will be the widely employed one. Histogram Equalization approach flattens your histogram and stretches your dynamic array of intensity values when using the cumulative thickness function. Nonetheless, there tend to be major bring backs throughout Histogram Equalization [9] especially when implemented in order to process electronic images. First of all, HE transforms the histogram from the input image right uniform histogram by distributing the full range regarding gray ranges uniformly in the histogram of an image, with some sort of mean value that is certainly in the heart of gray stage range. Keeping that in mind, the necessarily mean brightness from the output image is always at the middle - or next to it in the case of discrete rendering. In the situation of graphics with higher and small mean lighting value, there is a significant change within the view from the enhanced photograph. Secondly, histogram equalization performs the enhancement using the global content from the image. To put it differently, histogram equalization best parts the is bordered by and perimeters between various objects, but may reduce the local information on these physical objects, and not adequate regarding local development. Another consequence just for this mergence will be the production regarding over development and saturation artifacts [10].

Comparison advancement practices used commonly throughout picture control. One of the very most popular automatic procedures may be histogram equalization (HE). Out of your five sensory faculties sight, studying, feel, stench and design which individuals use in order to comprehend their own environment, sight would be the many highly effective. Obtaining and examining artwork types a substantial perhaps the routine cerebral activity of individuals throughout their own getting existence. In fact, more when compared with 99\% from the game from the human head is connected to control photos through the visible cortex. That is less successful if your distinction features range through the image. Versatile Histogram Equalization (AHE) overcomes that drawback by generating your mapping for each pixel through the histogram in an exceedingly surrounding screen. In long term, various design of photos will be taken probably in a variety of time period and utilize Versatile Histogram Equalization (AHE) and compare histogram equalization regarding images [11].

\subsection{Image Enhancement}

Picture advancement is just in regards to the easiest and many attractive elements of electronic photo processing. Fundamentally, thinking behind growth practices must be to create out detail that is obviously obscured or perhaps to identify certain alternatives that come with fascination within the image. The common example of growth where the distinction of a picture will be improved once and filtration it to remove the sounds "it seems better. " It is essential bear in mind that advancement is really a very subjective section of picture control. Improvement throughout quality of such degraded photos is possible by hiring program regarding advancement process [12].

\subsection{Adaptive Histogram Equalization method}

That is an expansion in order to traditional Histogram Equalization process. It enhances the proper execution a distinction of artwork by changing the values within the depth photograph. The AHE process might be understood in a variety of ways. Available together perception your histogram regarding gray stages (GL's) within the result may be maximally dark shade; if it's the conventional value throughout its monitor the output is $50 \%$ gray's monitor around every pixel may be generated primary. The cumulative publishing of GL's, this is actually the cumulative sum in the histogram [13]

\section{COLOR AND ILLUMINATION DISTRIBUTION}

Shade standing methods utilize data furnished by picture pixels to perform light appraisal. These pixels contain both spatial and shade details. Lately it is revealed where spatial data does not provide any added data with regards to light evaluation that can't be purchased straight through the color publishing [14]. Near the color publishing of particular individual photos, another supply of data which you should use in light opinion would be the circulation regarding possible light colors. For the successful chromatic edition, just your direction from the three-element vector is very important, while it's amplitude may be disregarded. That properly reduces your dimension as opposed to applying many three RGB components to cause it out the light supply, only a couple chromaticity components may be utilized. For some kind of shade explained featuring a RGB substances, the $\mathrm{h}$, and $\mathrm{n}$ chromaticity substances are determined these:

$$
\mathrm{r} *=\frac{r}{r=g=b}, \quad \mathrm{~g} *=\frac{g}{r=g=b}, \quad \mathrm{~b} *=\frac{b}{r=g=b}
$$

The habits of real-world illuminations are so apparent by while using the shade consistency standard datasets. Scenes associated with images in these datasets include calibration items applied to calculate your world light, that will be then presented as ground-truth illumination for each and every image when i. e. for each and every image the merit of elizabeth is properly known. While testing a way that will make an effort to determine elizabeth, your calibration objects are initial masked out, the strategy estimates your illumination elizabeth to the image landscape, and next that evaluation is set alongside the ground-truth light. The biggest widely obtainable shade consistency dataset could be the GreyBall dataset [15] which consists of 11346 photographs and groundtruth light elizabeth for all of them Because pretty much every camera uses its own RGB space that is unquestionably transformed with a standard RGB space later immediately after reaching coloring constancy, that demonstrates the relationship of light chromaticity components is as well as the applied RGB space. The exhibited persistence allows us to believe that typically it is enough to obtain the light just along only one line instead of looking due to it in the whole chromaticity planes. 


\section{Color Cat Approach}

It is really a novel in essence and accurate-based technique based on applying increased shade histogram charges as functions and regarding the uniformity regarding the probable gentle values. The approach is classified Color cat (CC) along with yes objective outperforms the majority of the different strategies on the subject of precision along with computation value hence mixing likely the most beneficial capabilities regarding just about every primary color constancy technique teams [16].

\section{RESULTS AND DISCUSSIONS}

Test Setup: - Every single child apply your proposed criteria, design and also implementation has been done in MATLAB utilizing image handling toolbox. Outcome showed which the proposed strategy gives increased results than the present approaches.

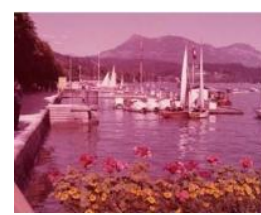

(a) input picture

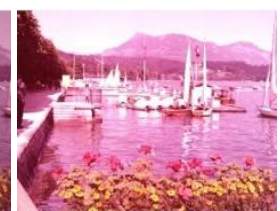

(b) existing technique

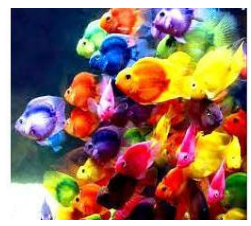

(c) Proposed Color

Cat method

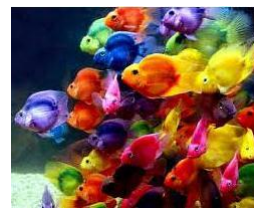

(a) input picture (b) existing

technique

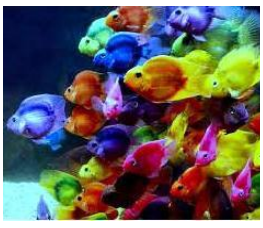

(c) Proposed Color Cat method
Fig: 2 : Experimental Results

\section{PERFORMANCE EVALUATION}

That area contains the mix validation between present and in the offing techniques. Some well-known picture performance parameters for electric pictures have been selected showing that the performance of the in the offing algorithm is very a lot better than the existing methods.

\subsection{Structure Similarity Index Matric}

It is showing the quantized evaluation of the organized likeness index metric. As organized likeness index metric need to increase which means planned algorithm is showing the greater effects than the available practices as SSIM has maximum value in every case.

Table 1: SSIM

\begin{tabular}{|c|l|l|}
\hline $\begin{array}{c}\text { INPUT } \\
\text { IMAGES }\end{array}$ & $\begin{array}{c}\text { EXISTING } \\
\text { RESULT }\end{array}$ & $\begin{array}{c}\text { PROPOSED } \\
\text { RESULT }\end{array}$ \\
\hline 1. & 0.6201 & 0.8255 \\
\hline 2. & 0.8665 & 0.9528 \\
\hline 3. & 0.7070 & 0.8049 \\
\hline 4. & 0.5113 & 0.9143 \\
\hline 5. & 0.4390 & 0.9162 \\
\hline
\end{tabular}

\begin{tabular}{|l|l|l|}
\hline 6. & 0.5083 & 0.9639 \\
\hline 7. & 0.5836 & 0.6662 \\
\hline 8. & 0.5529 & 0.9312 \\
\hline 9. & 0.6298 & 0.8325 \\
\hline 10. & 0.7086 & 0.9927 \\
\hline 11. & 0.6551 & 0.7408 \\
\hline 12. & 0.5524 & 0.9853 \\
\hline 13. & 0.7275 & 0.9679 \\
\hline 14. & 0.6344 & 0.9655 \\
\hline 15. & 0.3979 & 0.9978 \\
\hline
\end{tabular}

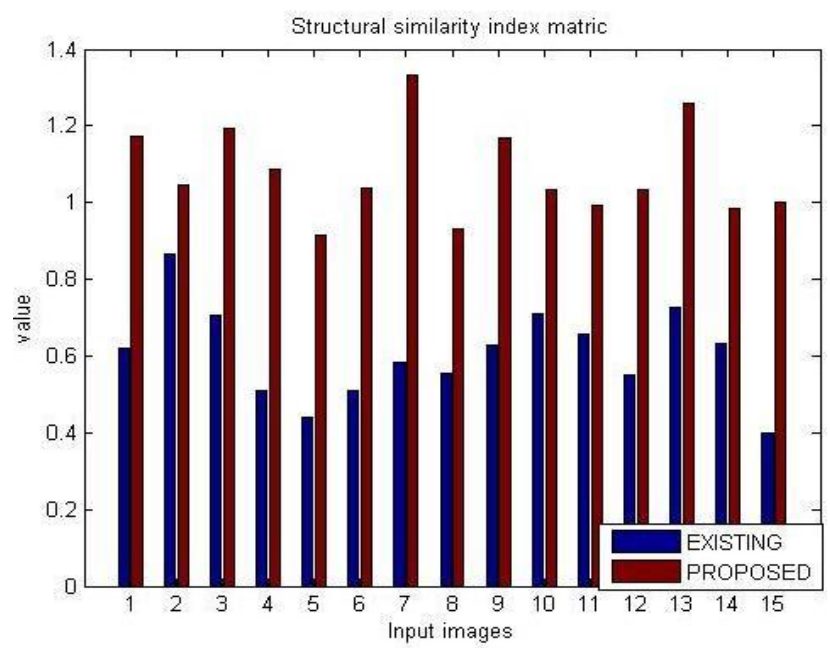

Fig: 3 : SSIM Graph.

\subsection{Median Angular Error}

It reflects the effect of light on a given image, so need to minimize the light on the image.

Table 2 Median Angular Error

\begin{tabular}{|c|l|l|}
\hline $\begin{array}{c}\text { INPUT } \\
\text { IMAGES }\end{array}$ & $\begin{array}{c}\text { EXISTING } \\
\text { RESULT }\end{array}$ & $\begin{array}{c}\text { PROPOSED } \\
\text { RESULT }\end{array}$ \\
\hline 1. & 1.5917 & 1.4127 \\
\hline 2. & 1.4588 & 1.3209 \\
\hline 3. & 1.5432 & 1.4788 \\
\hline 4. & 1.6548 & 1.4706 \\
\hline 5. & 1.6485 & 1.4078 \\
\hline 6. & 1.6065 & 1.4338 \\
\hline 7. & 1.6313 & 1.5145 \\
\hline 8. & 1.6070 & 1.4271 \\
\hline 9. & 1.6189 & 1.3998 \\
\hline 10. & 1.5229 & 1.4789 \\
\hline 11. & 1.4672 & 1.4333 \\
\hline 12. & 1.5727 & 1.4917 \\
\hline 13. & 1.5676 & 1.4919 \\
\hline 14. & 1.5898 & 1.3222 \\
\hline 15. & 1.6774 & 1.4185 \\
\hline
\end{tabular}




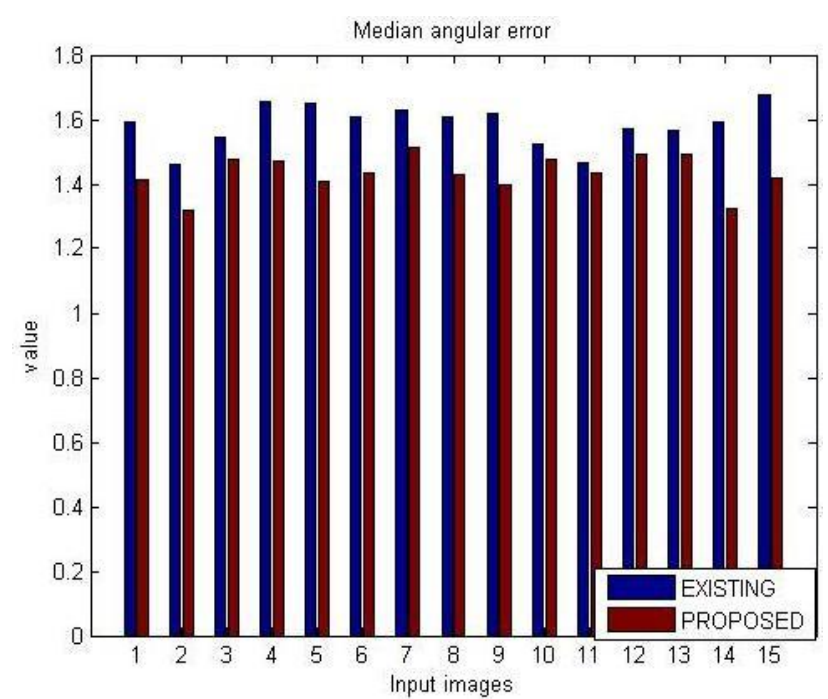

Fig: 4 : Median Angular Error Graph

\subsection{Bit Error Rate}

The touch problem rate (BER) may be the percentage of bits that have mistakes in accordance with the sum total number of bits received in a transmission. In electronic sign, how many touch mistakes is how many received items of a data flow over a transmission station which have been improved due to noise, disturbance, distortion or touch synchronization errors. The touch problem rate or touch problem rate (BER) is how many touch mistakes split by the sum total number of transferred bits throughout a studied time interval. shows the comparable examination of the Touch Mistake Rate (BER). BER needs to minimum for the proposed algorithm than present techniques. As shown in the table the outcome for proposed algorithm are minimum in every case. Therefore proposed algorithm offers better effects than present techniques.BitErrorRate $=100 \times(1-$ balanced classification rate)

Table3 Bit error rate

\begin{tabular}{|c|l|l|}
\hline $\begin{array}{c}\text { INPUT } \\
\text { IMAGES }\end{array}$ & $\begin{array}{c}\text { EXISTING } \\
\text { RESULT }\end{array}$ & $\begin{array}{c}\text { PROPOSED } \\
\text { RESULT }\end{array}$ \\
\hline 1. & 0.0159 & 0.0141 \\
\hline 2. & 0.0146 & 0.0123 \\
\hline 3. & 0.0154 & 0.0148 \\
\hline 4. & 0.0165 & 0.0147 \\
\hline 5. & 0.0165 & 0.0141 \\
\hline 6. & 0.0161 & 0.0143 \\
\hline 7. & 0.0163 & 0.0151 \\
\hline 8. & 0.0161 & 0.0143 \\
\hline 9. & 0.0162 & 0.0140 \\
\hline 10. & 0.0152 & 0.0148 \\
\hline 11. & 0.0147 & 0.0143 \\
\hline 12. & 0.0157 & 0.0149 \\
\hline 13. & 0.0157 & 0.0149 \\
\hline 14. & 0.0159 & 0.0132 \\
\hline 15. & 0.0168 & 0.0142 \\
\hline
\end{tabular}

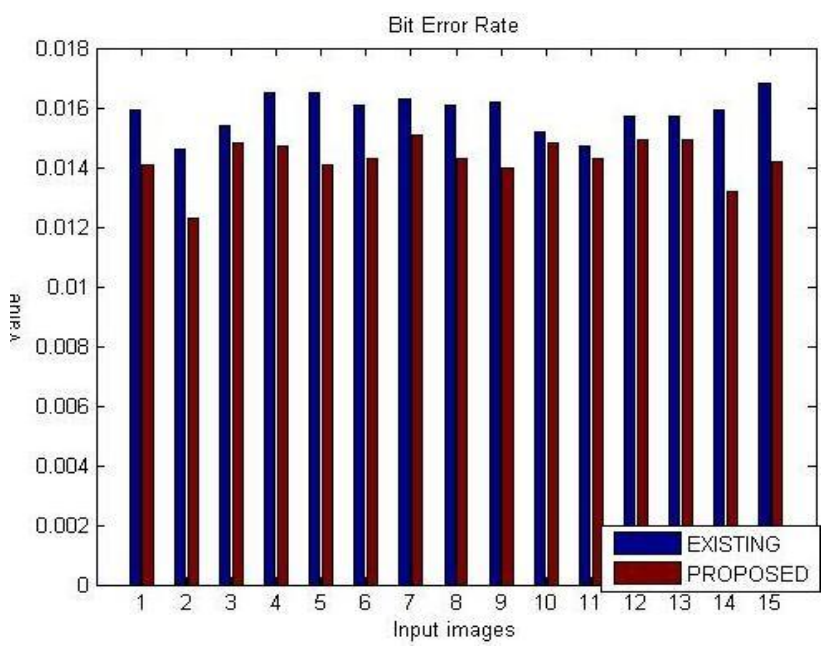

Fig: 5 : Bit Error Rate Graph

\section{CONCLUSION}

The new approaches for color constancy have been considered for evaluation purpose. The connected solutions to color constancy display that the use of adaptive histogram change is dismissed in $\mathrm{CC}$ but their use may reduce the unequal illuminate dilemma of color constancy. Along with CC algorithm may introduce minimal power image, therefore saturation weighting based calculations are anticipated to be incorporated with it. The use of hybrid tone constancy approach can be dismissed in the absolute most of active literature. Therefore to deal with this issue in that record a brand new incorporated color cat process is planned in that dissertation. The new process has used color normalization and saturation weighting as article handling of $\mathrm{CC}$ to minimize the result of unequal illuminate and bad brightness. The whole advantage has shown the effectiveness of the planned technique. That function hasn't considered the effectuation of numerous sort of appears on the insight image, therefore in not as distant potential a brand new approach can truly be planned to enhance the outcomes further.

\section{REFERENCES}

[1] K. Barnard. Improvements to gamut mapping colour constancy algorithms. ECCV, 2000.

[2] K. Barnard, L. Martin, A. Coath, and B. Funt. A comparison of computational color constancy algorithms: Experiments with image data. TIP, 2002.

[3] H. G. Barrow and J. M. Tenenbaum. Recovering Intrinsic Scene Characteristics from Images. Academic Press, 1978.

[4] S. Bianco and R. Schettini. Color constancy using faces. CVPR, 2012.

[5] D. H. Brainard and W. T. Freeman. Bayesian color constancy. JOSA A, 1997.

[6] G. Buchsbaum. A spatial processor model for object colour perception. Journal of the Franklin Institute, 1980.

[7] A. Chakrabarti, K. Hirakawa, and T. Zickler. Color constancy with spatio-spectral statistics. TPAMI, 2012.

[8] D. Cheng, D. K. Prasad, and M. S. Brown. Illuminant estimation for color constancy: why spatial-domain methods work and the role of the color distribution. JOSA A, 2014. 
[9] G. D. Finlayson, S. D. Hordley, and P. M. Hubel. Color by correlation: A simple, unifying framework for color constancy. TPAMI, 2001.

[10] D. A. Forsyth. A novel algorithm for color constancy. IJCV, 1990.

[11] P. Gehler, C. Rother, A. Blake, T. Minka, and T. Sharp. Bayesian color constancy revisited. CVPR, 2008.

[12] A. Gijsenij and T. Gevers. Color constancy using natural image statistics and scene semantics. TPAMI, 2011.

[13] A. L. Gilchrist. Seeing Black and White. Oxford University Press, 2006.

[14] J. van de Weijer, T. Gevers, and A. Gijsenij. Edge-based color constancy. TIP, 2007.

[15] G. D. Finlayson, M. S. Drew, and C. Lu, "Intrinsic images by entropy minimization," in Computer VisionECCV 2004. Springer, 2004, pp. 582-595.
[16] B. Maxwell, C. Smith, and R. Friedhoff, "Method and system for separating illumination and reflectance using a $\log$ color space," Oct. 18 2007, US Patent App. $11 / 403,719$

[17] Y. Bai and X. Zhang, "Transformations and White Point Constraint Solutions for a Novel Chromaticity Space," Apr. 18 2013, US Patent App. 13/342,873.

[18] B. Mazin, J. Delon, and Y. Gousseau, "Illuminant estimation from projections on the planckian locus," in Computer Vision-ECCV 2012. Workshops and Demonstrations. Springer, 2012, pp. 370-379.

[19] B. Zhang and A. Batur, "Method and apparatus for white balance," Jan. 28 2010, US Patent App. 12/510,853.

[20] B. Zhang, "Method and apparatus for improving the stability of histogram correlation algorithm," Sep. 10 2013, US Patent 8,532,381. 